\title{
Chronische Wunden und die Relevanz ihrer Behandlung
}

\author{
Gerhard Köveker Eike Sebastian Debus $^{\mathrm{b}}$ \\ a Städtisches Krankenhaus, Allgemeinchirurgie, Sindelfingen,

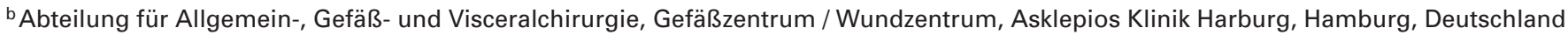

In Deutschland leiden etwa 3 Millionen Menschen an chronischen Wunden. Dies entspricht fast $4 \%$ der Bevölkerung. Das Ulcus cruris venosum stellt mit $57-80 \%$ aller chronischen Ulzera die häufigste Ursache nicht spontan abheilender Wunden dar. Analysen für das ehemalige Westdeutschland ergaben eine jährliche volkswirtschaftliche Gesamtbelastung in Höhe von 2-3 Milliarden DM allein für die venösen Ulzera cruris. Die Altersentwicklung in Deutschland zeigt in den vergangenen Jahren einen stetig steigenden Anteil der über 65-Jährigen bei stagnierender bzw. rückläufiger Geburtenzahl. Es ist zu erwarten, dass die zunehmende Zahl älterer Menschen auch eine Zunahme von Problemwunden zur Folge haben wird. Die Beschäftigung mit der Wundbehandlung ist vor diesem Hintergrund von besonderer Wichtigkeit. Neben dieser nicht unerheblichen sozialmedizinischen Bedeutung hat eine chronische, nicht heilende Wunde jedoch auch Rückwirkungen auf den Alltag und die Lebensqualität jedes Betroffenen. Untersuchungen hierzu machen insgesamt deutlich, dass eine chronische Wunde für den Betroffenen eine erhebliche Einschränkung bedeutet. Angesichts der oft langwierigen Behandlungen, die eine deutliche Verbesserung der Lebensqualität bewirken können, sind neben der Beschleunigung der Abheilung auch Fragen des Patientenkomforts, der Schmerzhaftigkeit der Wunde und des Verbandwechsels von besonderer Bedeutung. Wunden sind umschriebene Gewebeläsionen, deren Behandlung und Heilungsaussichten abhängig sind von der Wundentstehung und der Wundätiologie. Neben dem fortgeschrittenen Alter gibt es auch systemische Erkrankungen, die sich negativ auf die Wundheilung auswirken und daher suffizient mitbehandelt werden müssen (z.B. Niereninsuffizienz, Herzinsuffizienz, Diabetes mellitus, Mangelernährung, Immunsuppression). Notfallsituationen (Ileus, Perforation), adjuvante Therapiemaßnahmen wie Radio-/Chemotherapie und schwere Komorbidität sind hingegen Beispiele für nicht beeinflussbare systemische Faktoren, die zu Störungen der Wundheilung führen können.
Obwohl seit Jahrzehnten wissenschaftlich begründet, hat sich die feuchte Wundbehandlung erst in den letzten Jahren durchgesetzt. Semiokklusive und okklusive Wundauflagen, die in den letzten Jahren von der Industrie entwickelt worden sind, ermöglichen erstmals eine phasengerechte Wundbehandlung. Ihr interaktives Therapieprinzip führt in erster Linie zu einer Verbesserung des Wundmilieus.

Als enttäuschend sind die bisherigen klinischen Erfahrungen einzustufen, mit lokal applizierten Wachstumsfaktoren aktiv in den Wundheilungsprozess einzugreifen. Nichtsdestotrotz scheint der Transfer molekularbiologisch basierter Therapieansätze in die klinische Anwendung nur noch eine Frage der Zeit zu sein.

Die nächstliegenden Aufgaben sind sicher in der Verbesserung der Versorgungsstrukturen zu suchen, was vor dem Hintergrund der sektoralen Versorgung mit besonderen Schwierigkeiten verbunden ist. Die Bildung von Wundzentren stellt eine aktuelle und interessante Herausforderung im Rahmen der kommenden Neuordnung des Gesundheitswesens in Deutschland dar. Die Implementierung macht eine Neuordnung bisheriger Abteilungsstrukturen erforderlich, die sich sowohl in medizinischen Abläufen als auch in der betriebswirtschaftlichen Organisationsstruktur niederschlägt. Diese Organisationsstruktur optimiert die Prozessabläufe, so dass das Management insgesamt patientengerechter gestaltet werden kann. Die Zentrumsbildung stärkt die Kompetenz in der medizinischen Versorgung, wodurch eine Steigerung der Qualität und damit eine Verbesserung der (außer- und innerbetrieblichen) Akzeptanz erreicht werden kann. Mehrfach konnte gezeigt werden, dass durch Zentrumsbildung eine schnellere Rehabilitation und weniger deletäre Eingriffe (d.h. MajorAmputationen) möglich sind. Hierdurch wird in vielen Fällen die Wiedereingliederung der Betroffenen in die Gesellschaft und in das Berufsleben möglich - ein Effekt, der auch in der internationalen Literatur nachgewiesen werden konnte.

\begin{tabular}{ll}
\hline KARGER & @ 2006 S. Karger GmbH, Freiburg \\
Fax +49761 4520714 & Accessible online at: \\
$\begin{array}{l}\text { E-mail Information@Karger.de } \\
\text { www.karger.com }\end{array}$ & www.karger.com/cga
\end{tabular}


Die Bedeutung der Wundheilung ist allerdings nicht auf Krankheitsbilder mit Läsionen von Haut und Weichteilen begrenzt. Im Gefäßsystem führen gestörte Reparationsmechanismen häufig zu einer überschießenden Gewebereaktion, wie wir sie als Intimahyperplasie, nach endovaskulären Verfahren aber auch an Anastomosen beobachten.

Wundheilungsstörungen nach aseptischen bzw. bedingt kontaminierten Eingriffen werden zunehmend in Qualitätssicherungssystemen erfasst und dienen als Ersatzparameter der chirurgischen Qualität. So genannte OP-Gebiet-konforme Wundheilungsstörungen (SSI, surgical site infection) haben auch ökonomische Bedeutung, sie repräsentieren fast $40 \%$ aller nosokomialen Infektionen, verlängern die Dauer des Krankenhausaufenthalts um durchschnittlich 7 Tage und tragen damit zu einer erheblichen Kostensteigerung bei.

Im vorliegenden Heft haben wir versucht, unterschiedliche Aspekte der Wundheilung zu berücksichtigen, haben uns aber, um der Komplexität dieses Themas Rechnung zu tragen, auf Läsionen der Haut und Weichteile beschränkt. Es ist gelungen, kompetente und erfahrene Autoren für dieses Schwerpunktheft $\mathrm{zu}$ gewinnen, das die Bedeutung dieses lange von der klinischen Medizin vernachlässigten Themas aufzeigt. Wir wünschen Ihnen viel Spaß beim Lesen! 